\title{
Patient Diversity Is Our Reality: the Covariates Matter
}

\author{
Charles N. Cornell, MD
}

Received: 17 May 2019/Accepted: 17 May 2019/Published online: 4 June 2019

(C) Hospital for Special Surgery 2019

It is my pleasure to bring you the latest issue of $H S S$ Journal®. This issue presents several articles on the hand and upper extremity, one in particular that should catch the interest of shoulder surgeons. For reconstructive surgery on the shoulder, general anesthesia is often required, and patients are often placed in the beach chair position. Many anesthesiologists have suggested that general anesthesia applied in this position increases risk of cerebral oxygen desaturation. In "Cerebral Oxygenation in the Sitting Position Is Not Compromised During Spontaneous or PositivePressure Ventilation" (https://doi.org/10.1007/s11420-0189642-4), Ya Deau and his colleagues investigated the cerebral blood oxygenation of 25 patients undergoing total shoulder arthroplasty using general anesthesia in the beach chair position. This prospective, observational study found no cerebral desaturation events in this cohort, providing supportive evidence to the greater anesthesia community that cerebral oxygenation and perfusion can be safely maintained in the beach chair position.

Perhaps the most impactful article in this issue is "Total Hip Arthroplasty Performed for Coxarthrosis Preserves Long-Term Physical Function: A 40-Year Experience" (https://doi.org/10.1007/s11420-019-09676-0). I must disclose that I am a co-author, but the senior and lead author is Philip D. Wilson Jr., MD, who died in 2016. This article typifies Dr. Wilson's important legacy as a pioneer of the science and practice of total hip arthroplasty (THA). As opposed to prior long-term follow-up reports that focused

\footnotetext{
C. N. Cornell, MD $(\triangle)$

Hospital for Special Surgery,

535 East 70th Street,

New York, NY 10021, USA

e-mail: cornellc@hss.edu

C. N. Cornell, MD

Weill Cornell Medicine,

1300 York Avenue,

New York, NY 10065, USA
}

only on radiographic findings or revision rates, this study confirms the long-term benefit of THA by examining clinical outcomes, such as pain level, walking ability, and range of motion. One important study conclusion is that the improvement in hip function THA provides is enduring even if revision surgery is needed. Most patients in our case series who required revision regained their initial post-THA function - restoring for them the benefits of the procedure.

Another significant finding of that study was that patient characteristics and covariates greatly influence the outcomes of THA. Traditionally, most clinical reports in orthopedics have used linear class models that report on patients as a homogeneous population. Recently, understanding on how covariates influence outcomes has grown, changing how observational studies are conducted. In our study, the statistician, Yuo-yu Lee, MS, applied a latent class mixed model approach. This approach predicts that subgroupings of patients with similar characteristics and outcomes can be identified within the study population. In our study, the model identified four subgroupings of patients with similar characteristics and outcomes. When the data were analyzed as a whole, the results were ambiguous, but when the latent class mixed model was applied, the results were dramatic. For example, one subgrouping, the elderly class (mean age, 62 years), showed significant initial benefits of THA that eventually declined, about 20 years after the primary surgery.

Surgeons must bear in mind that the risks and benefits of a surgical intervention will not be evenly distributed across a population. Our patient populations are not monoliths. Individual characteristics and covariates strongly influence outcomes and must be factored into analyses of clinical results. Reporting on means is no longer acceptable in observational studies, and such studies must be powered with sufficient numbers to allow for multifactorial analysis. This emerging principle has been cited in numerous clinical fields in which traditional reporting has led to changes in practice, only to be proven misleading when the heterogeneity of the patient population was properly analyzed [1-3]. Patient diversity is our reality, and the covariates definitely matter. 
Compliance with Ethical Standards

Conflict of Interest: Charles N. Cornell, MD, reports personal fees from Exactech, Inc., outside the submitted work.

\section{Human/Animal Rights: N/A}

Informed Consent: N/A

Required Author Forms Disclosure forms provided by the authors are available with the online version of this article.

\section{References}

1. Krinsley JS. The long and winding road toward personalized glycemic control in the critically ill. J Diabetes Sci Technol. 2018;12(1):26-32.

2. Mahieu MA, Strand V, Simon LS, Lipsky PE, Ramsey-Goldman R. A critical review of clinical trials in systemic lupus erythematosus. Lupus. 2016;25(10):1122-1140.

3. Santhakumaran S, Gordon A, Prevost AT, O'Kane C, McAuley DF, Shankar-Hari M. Heterogeneity of treatment effect by baseline risk of mortality in critically ill patients: re-analysis of three recent sepsis and ARDS randomised controlled trials. Crit Care. 2019;23(1):156. 